\title{
Consumo de Alimentos Ultraprocessados por Mulheres Sobreviventes do Câncer de Mama
}

doi: https://doi.org/10.32635/2176-9745.RBC.2020v66n3.1092

\author{
Intake of Ultra-Processed Foods by Women Surviving Breast Cancer \\ Consumo de Alimentos Ultra Procesados por Mujeres Supervivientes del Cáncer de Mama
}

Julianne do Nascimento Sales'; Manuella Cunha Barbosa²; Ilana Nogueira Bezerra³; Sara Maria Moreira Lima Verde

RESUMO

Introduçáo: A elevada prevalência de câncer de mama no Brasil em paralelo ao aumento no consumo de alimentos ultraprocessados sugere relação estreita entre esses fatores. Objetivo: Avaliar o consumo de alimentos ultraprocessados em mulheres sobreviventes do câncer de mama. Método: Estudo transversal com 100 mulheres com câncer de mama acompanhadas em centro de tratamento oncológico. Utilizando um questionário de frequência alimentar, os itens alimentares consumidos foram agrupados em in natura, processados e ultraprocessados, conforme classificação NOVA. A contribuição energética de cada grupo de alimento deu-se pela razão entre caloria proveniente do grupo e caloria total. As pacientes foram categorizadas em elevado e baixo consumo de ultraprocessados e as diferenças entre os dois grupos (variáveis categóricas) foram testadas por $\mathrm{X}^{2}$ de Pearson. A relaçáo entre a ingestáo de calorias provenientes de ultraprocessados e a ingestão de energia e de nutrientes específicos foi baseada em modelos de regressão linear brutos e ajustados por idade, escolaridade e índice de massa corporal. Resultados: Das calorias ingeridas pelas pacientes, 27,1\% eram de ultraprocessados. As com alto consumo de ultraprocessados tinham menor ingestáo de proteínas $(\mathrm{p}=0,0372)$ e fibras $(\mathrm{p}=0,0458)$ e maior de gordura poli-insaturada ( $\mathrm{p}=0,0019)$ e sódio $(\mathrm{p}=0,0068)$. O consumo de ultraprocessados implicou em menor ingestấo de in natura e maior de sódio, gordura total e de suas fraçóes $(\mathrm{p}<0,05)$. Conclusáo: Mulheres sobreviventes do câncer de mama têm um terço da sua alimentaçáo composto por ultraprocessados associados à redução no consumo de in natura, proteínas e fibras.

Palavras-chave: Consumo de Alimentos; Alimentos Industrializados; Qualidade dos Alimentos; Neoplasias da Mama; Sobrevivência.

\section{ABSTRACT}

Introduction: High prevalence of breast cancer in Brazil along with the increase in intake of ultra-processed foods suggests a narrow relation between these two factors. Objective: To evaluate the intake of ultra-processed foods in women surviving breast cancer. Method: Cross-sectional study with 100 women with breast cancer followed at an oncology treatment center. Based in a food frequency questionnaire, foods items ingested were grouped in in natura, processed and ultra-processed, according to NOVA classification. The energetic contribution of each food group was given by the ratio between calories from the group and total calory. Patients were categorized in high and low intake of ultra-processed and the differences between the two groups (categorical variables) were tested by Pearson's $\mathrm{X}^{2}$ test. The relationship between calories intake from ultra-processed foods and the intake of energy and specific nutrients was based inn linear regression models adjusted per age, education and body mass index. Results: Of the calories ingested by the patients, $27.1 \%$ were ultra-processed foods. Those with high ingestion of ultra-processed foods had lower intake of protein $(\mathrm{p}=0.0372)$ and fibers $(\mathrm{p}=0.0458)$ and higher intake of polyunsaturated fat $(\mathrm{p}=0.0019)$ and sodium $(\mathrm{p}=0.0068)$. The ingestion of ultra-processed foods was related to lower intake of in natura foods and higher intake of sodium, total fat and its fractions $(\mathrm{p}<0.05)$. Conclusion: Women who survived breast cancer had one third of their diet formed by ultra-processed foods associated with reduced intake of in natura, proteins and fibers.

Key words: Food Consumption; Industrialized Foods; Food Quality; Breast Neoplasms; Survivorship.

\section{RESUMEN}

Introducción: La elevada prevalencia de cáncer de mama en Brasil ha aumentado junto con el aumento del consumo de alimentos ultraprocesados. Objetivo: Evaluar el consumo de alimentos ultraprocesados de mujeres supervivientes del cáncer de mama. Método: Estudio transversal con 100 mujeres con cáncer de mama asistidas en un centro de tratamiento oncológico. Utilizando un cuestionario de frecuencia alimentaria, se ha dividido los ítems consumidos en naturales, procesados y ultraprocesados según la clasificación NOVA. La contribución energética de cada grupo de alimento se dio por la división entre la caloría del grupo y la total. Se ha categorizado a las pacientes en alto y bajo consumo de ultraprocesados y se ha testado las diferencias entre los dos grupos mediante la prueba de chi-cuadrado. Se ha basado en modelos de regresión lineal ajustados por edad, educación e índice de masa corporal para la relación entre la ingesta de calorías de los alimentos ultraprocesados y de energía y nutrientes específicos Resultados: De las calorías ingeridas por las pacientes, 27,1\% fueran de ultraprocesados. Las de alto consumo de ultraprocesados tenían menos ingesta de proteínas $(\mathrm{p}=0,0372)$ y fibras $(\mathrm{p}=0,0458)$ y mayor ingesta de grasa poli-insaturada $(\mathrm{p}=0,0019)$ y sodio $(\mathrm{p}=0,0068)$. El consumo de ultraprocesados ha llevado a menos ingesta de alimentos naturales y mayor ingesta de sodio, de grasa total y de sus fracciones $(\mathrm{p}<0,05)$. Conclusión: Las mujeres supervivientes del cáncer de mama tuvieron un tercio de su alimentación formado por ultraprocesados asociados a la disminución del consumo de alimentos naturales, proteínas y fibras.

Palabras-clave: Consumo de Alímentos; Alimentos Industrializados; Calidad de los Alimentos; Neoplasias de la Mama; Supervivencia.

'Nutricionista. Residente no Serviço de Nutrição do Hospital Haroldo Juaçaba. Instituto do Câncer do Ceará (ICC). Fortaleza (CE), Brasil. Orcid iD: https://orcid. org/0000-0001-8426-5345

${ }^{2}$ Nutricionista. Mestranda do Programa de Pós-graduação em Nutrição e Saúde da Universidade Estadual do Ceará (UECE). Fortaleza (CE), Brasil. Orcid iD: https:// orcid.org/0000-0002-7245-6723

${ }^{3}$ Nutricionista. Pesquisadora do Programa de Pós-graduação em Nutrição e Saúde. UECE. Fortaleza (CE), Brasil. Orcid iD: https://orcid.org/0000-0002-2072-0123 ${ }^{4}$ Nutricionista. Pesquisadora do Programa de Pós-graduação em Nutrição e Saúde. UECE. Fortaleza (CE), Brasil. Orcid iD: https://orcid.org/0000-0002-7733-0214 Endereço para correspondência: Sara Maria Moreira Lima Verde. Av. Dr. Silas Munguba, 1700 - Campus do Itaperi. Fortaleza (CE), Brasil. CEP $60741-000$. E-mail: sara.maria@uece.br 


\section{INTRODUÇÃO}

O consumo de ultraprocessados tem sido amplamente investigado em sua relação com obesidade e outras doenças crônicas ${ }^{1}$. No câncer, o terceiro relatório do World Cancer Research Fund (WCRF) ${ }^{2}$ coloca como forte evidência para o risco de câncer de cólon o consumo de ultraprocessados. Apesar de as evidências dessa relação ainda não serem suficientes para o câncer de mama ${ }^{3,4}$, uma metanálise recente indica que o aumento no consumo de ultraprocessados está associado ao aumento no risco de câncer de mama ${ }^{5}$.

Essa associação entre ultraprocessados e câncer de mama pode ser explicada de forma indireta pelo fato de esses alimentos serem de elevada densidade energética e baixo valor nutricional ${ }^{6}$, contribuindo para obesidade e excesso de tecido adiposo ${ }^{7}$, postos como fatores de risco para a doença ${ }^{8}$. Além disso, os alimentos ultraprocessados têm em sua formulação componentes que favorecem o processo de carcinogênese ${ }^{9}$ ou aditivos alimentares que funcionam como disruptores carcinogênicos por sua exposição desde a puberdade ${ }^{10}$.

Para além do risco de desenvolver a doença, a elevada prevalência de sobreviventes do câncer de mama com mais de 6,8 milhóes de mulheres no mundo ${ }^{11}$ impulsiona o cuidado com a alimentação por ser um fator de risco modificável ${ }^{12}$ e contribuir para minimizar a recidiva da doença, em especial a recidiva tardia que acontece cinco a 20 anos após o diagnóstico ${ }^{13}$. Nesse contexto, o Instituto Nacional de Câncer José Alencar Gomes da Silva (INCA) expressa sua preocupação com a obesidade e alimentação inadequada da população brasileira nas últimas décadas e a necessidade de mitigar essa epidemia para prevenir novos casos da doença ${ }^{14}$.

Diante do exposto, o presente estudo verificou o consumo de alimentos segundo a extensão e o propósito do seu processamento entre mulheres sobreviventes do câncer de mama, a fim de identificar a contribuição dos alimentos ultraprocessados e suas associaçóes com a ingestão de nutrientes específicos.

\section{MÉTODO}

Trata-se de um estudo transversal realizado com mulheres sobreviventes ${ }^{15}$ do câncer de mama $(n=100)$, atendidas no Centro Regional Integrado de Oncologia, referência para o tratamento oncológico na cidade de Fortaleza - CE, no Nordeste do Brasil. Selecionadas de forma consecutiva e não probabilísticas, as participantes eram maiores de 20 anos, não tinham história prévia de outra neoplasia, não estavam em tratamento quimioterápico e não tinham recebido orientação nutricional.
A coleta de dados foi realizada por pesquisadores treinados em entrevista direta e estudo dos prontuários. Foram colhidas informações sociodemográficas (idade, anos de estudo, estado civil, renda mensal em salários mínimos), estadiamento clínico, medidas antropométricas [peso atual (PA-Kg), altura $(\mathrm{m})$ e circunferência da cintura (CC-cm)] e consumo alimentar.

O PA e a altura foram aferidos em balança plataforma Plenna ${ }^{\oplus}$ com capacidade máxima de $150 \mathrm{~kg}$ e precisão de $0,1 \mathrm{~kg}$, com estadiômetro acoplado com a precisão 0,1 $\mathrm{cm}$. Para o PA, as pacientes estavam com roupas leves e descalças; para a altura, seguiu-se a posição de Frankfurt ${ }^{16}$. A partir desses parâmetros, foi calculado o índice de massa corporal (IMC) (peso/altura²) para diagnóstico nutricional, segundo a classificação da Organização Mundial da Saúde (OMS) ${ }^{17}$ para mulheres adultas e de Lipschitz ${ }^{18}$ para idosas.

A CC foi medida com fita métrica flexível e inelástica com precisão de $0,1 \mathrm{~cm}$ no ponto médio entre a crista ilíaca e a última costela ${ }^{19}$, sendo definido como ponto de corte o valor $\geq 88 \mathrm{~cm}^{19,20}$, indicativo de risco elevado para doenças cardiometabólicas.

Para conhecer o consumo alimentar das pacientes, utilizou-se o questionário quantitativo de frequência alimentar $(\mathrm{QQFA})^{21}$, validado para as mulheres do Nordeste brasileiro, com 68 itens alimentares, aplicado por entrevistador treinado. $\mathrm{O}$ uso de registros fotográficos e kits com medidas caseiras (pratos, xícaras, copos e talheres) características da região foi utilizado para estimar a quantidade das porçóes consumidas. Atribuiu-se peso 1 para a frequência de consumo de uma vez por dia, e foram considerados pesos proporcionais para as demais respostas de frequência. Assim, a frequência de consumo para cada um dos itens alimentares incluídos no QQFA foi transformada em frequência diária. Em seguida, foi calculado o consumo alimentar diário de cada paciente em gramas ou mililitros e posteriormente em calorias, macronutrientes (carboidrato, proteína, lipídeo, gordura saturada, gorduras poli e monoinsaturada, colesterol, fibra) e micronutriente (sódio), utilizando a Tabela Brasileira de Composição de Alimentos (TACO), a USDA Nutrient Database for Standard Reference e a Tabela de Composição Nutricional dos Alimentos Consumidos no Brasil do Instituto Brasileiro de Geografia e Estatística (IBGE).

Cada item do QQFA foi caracterizado de acordo com a extensão e o propósito do processamento a que foi submetido, considerando a classificação NOVA, proposta por Monteiro et al. ${ }^{22}$, sendo categorizado em um dos seguintes grupos: alimentos in natura ou minimamente processados e ingredientes culinários processados, alimentos processados e alimentos ultraprocessados. Em seguida, estimaram-se as calorias provenientes de cada 
grupo para cada participante, somando-se a ingestáo relativa de energia de cada item do QQFA de acordo com sua classificação. A média da contribuição percentual de cada grupo de alimento, segundo o tipo de processamento na ingestáo calórica total, foi estimada a partir do cálculo da média das razóes (caloria proveniente do grupo de alimento/caloria total) de cada indivíduo.

As mulheres foram categorizadas em elevado e baixo consumo de alimentos ultraprocessados, considerando o percentil 75 e 25 de calorias provenientes dos alimentos ultraprocessados, respectivamente. As diferenças nas frequências das variáveis categóricas entre os dois grupos foram testadas a partir do teste do qui-quadrado de Pearson.

Para avaliar a relação entre a ingestão de calorias provenientes de ultraprocessados e a ingestão de energia (proveniente de alimentos in natura e de alimentos açucarados) e de nutrientes específicos (proteína, lipídeos, gorduras saturada, monoinsaturada, poli-insaturada, fibra e sódio), utilizaram-se modelos de regressão linear, sendo as calorias provenientes de ultraprocessados a variável independente. A ingestão de ultraprocessados foi ajustada pela ingestấo total de energia, usando-se o método do resíduo $^{23}$ e, uma vez que ela apresentou distribuição assimétrica, essa variável sofreu transformação logarítmica. Após ajuste pela energia, o exponencial do valor ajustado foi calculado para facilitar a interpretaçáo dos dados. Os modelos foram posteriormente ajustados por idade, escolaridade e IMC.

Consideraram-se, como alimentos açucarados, biscoito doce sem recheio, biscoito doce recheado; açúcar adicionado ao leite; iogurte com frutas; vitamina de leite ou leite batido com frutas; suco de laranja; suco natural de outras frutas; café com açúcar; chocolates, bombons e brigadeiro; doces de frutas; bolos e tortas; sorvete e refrigerante não dietético ou dietético.

Todas as análises estatísticas foram realizadas no programa SAS, versão 9.4, adotando significância de 5\%.

Este estudo foi aprovado pelo Comitê de Ética em Pesquisa da Universidade de Fortaleza (Unifor) sob o número de parecer 204/10 e a coleta de informaçóes iniciada após as pacientes assinarem o Termo de Consentimento Livre e Esclarecido (TCLE).

\section{RESULTADOS}

As pacientes tinham idade média de 50,9 ( $\mathrm{DP}=10,2)$ anos, $53 \%$ estavam na faixa etária $\geq 50$ anos e $10 \%$ delas eram idosas. A maioria tinha companheiro $(61,2 \%)$, estudou até o ensino fundamental $(71,6 \%)$, possuía renda mensal inferior a quatro salários mínimos $(63 \%$; $\mathrm{R} \$$ 622,00 - U\$311,74) e estava em estadiamentos clínicos mais avançados (III e IV) $(73,9 \%)$ (Tabela 1).
O IMC médio das participantes era $28,8 \mathrm{~kg} / \mathrm{m}^{2}$ (desvio-padrão - $\mathrm{DP}=4,5)$ e $32 \%$ estavam obesas. A média de CC era $98,6 \mathrm{~cm}(\mathrm{DP}=177,6)$ e $86,5 \%$ das pacientes estava com risco elevado para doença cardiometabólica (CC $\geq 88$ $\mathrm{cm})$. Com relaçáo ao consumo de ultraprocessados, os grupos mostraram perfis sociodemográfico, clínico e antropométrico semelhantes (Tabela 1).

O consumo calórico médio das participantes do estudo foi de $2.278,5 \mathrm{kcal}(\mathrm{DP}=862,5)$, das quais $27,1 \%$ proviam dos alimentos ultraprocessados, representados em sua maior parte por pães industrializados, biscoitos recheados, biscoitos doces e biscoitos salgados e por alimentos embutidos. Os alimentos in natura representaram 69,81\% das calorias consumidas, sendo a maior parte proveniente de carnes e ovos (13,56\%), seguida de frutas $(9,30 \%)$ e leite $(6,76 \%)$ (Tabela 2).

A Tabela 3 apresenta o consumo de macronutrientes (carboidrato, proteína e gordura), fibras e sódio de acordo com o maior ou menor quartil de consumo de ultraprocessados, em que as pacientes que consomem mais ultraprocessados têm menor ingestão de proteínas e fibras e maior consumo de gordura poli-insaturada e sódio.

A Tabela 4 mostra a relação entre a ingestão de ultraprocessados e a ingestáo de energia e de nutrientes específicos, na qual observa-se que o consumo de alimentos ultraprocessados implica em redução na ingestáo de calorias provenientes de alimentos in natura e no aumento de gordura total e de suas fraçôes $(\mathrm{p}<0,05)$. A ingestão de sódio também aumenta com o maior consumo de alimentos ultraprocessados $(\mathrm{p}<0,05)$. Essas relaçôes foram independentes da idade, escolaridade e do IMC das mulheres.

\section{DISCUSSÃO}

O presente estudo investigou o consumo de alimentos, segundo a extensão e o propósito de processamento por mulheres sobreviventes do câncer de mama e mostra que quase um terço das calorias ingeridas por essas pacientes é proveniente de alimentos ultraprocessados e que, quanto mais elevada essa ingestáo, menor é o consumo de alimentos in natura, proteínas e fibra e maior o de gordura total, suas fraçôes e sódio.

O consumo de ultraprocessados tem trazido preocupação quanto ao seu impacto no ganho excessivo de peso e no desenvolvimento de doenças crônicas não transmissíveis por favorecerem maior consumo de calorias e açúcar de adiçãa ${ }^{1,7}$. Os achados deste estudo fortalecem a necessidade de se ter um olhar especial para esse hábito alimentar entre mulheres sobreviventes do câncer de mama, uma vez que o maior consumo de calorias contribui 
Tabela 1. Tamanho da amostra, características sociodemográficas, nutricionais e clínicas, segundo o consumo de alimentos ultraprocessados. Fortaleza, Ceará, Brasil

\begin{tabular}{|c|c|c|c|c|c|}
\hline Variável & $\mathbf{N}$ & $\%$ & $\begin{array}{l}\text { Baixo consumo de } \\
\text { ultraprocessados }\end{array}$ & $\begin{array}{l}\text { Alto consumo de } \\
\text { ultraprocessados }\end{array}$ & p-valor \\
\hline \multicolumn{6}{|l|}{ Idade $(n=100)$} \\
\hline$<50$ anos & 47 & 56,0 & 52,0 & 60,0 & 0,572 \\
\hline$\geq 50$ anos & 53 & 44,0 & 48,0 & 40,0 & \\
\hline \multicolumn{6}{|l|}{ Estado civil $(n=98)$} \\
\hline Sem companheiro & 38 & 38,8 & 40,0 & 24,0 & 0,715 \\
\hline Com companheiro & 60 & 61,2 & 60,0 & 76,0 & \\
\hline \multicolumn{6}{|l|}{ Escolaridade $(n=95)$} \\
\hline Ensino fundamental & 68 & 71,6 & 80,0 & 72,0 & 0,822 \\
\hline Ensino médio e superior & 27 & 28,4 & 20,0 & 28,0 & \\
\hline \multicolumn{6}{|l|}{ Renda $(n=92)$} \\
\hline <4 salários mínimos & 58 & 63,0 & 58,3 & 63,6 & 0,946 \\
\hline$\geq 4$ salários mínimos & 34 & 37,0 & 41,7 & 36,4 & \\
\hline \multicolumn{6}{|c|}{ Estado nutricional $(n=100)$} \\
\hline Eutrofia & 20 & 20,0 & 28,0 & 12,0 & 0,100 \\
\hline Sobrepeso & 48 & 48,0 & 36,0 & 44,0 & \\
\hline Obesidade & 32 & 32,0 & 36,0 & 44,0 & \\
\hline \multicolumn{6}{|c|}{ Risco cardiometabólico $(n=96)$} \\
\hline Baixo/Moderado & 14 & 14,6 & 8,0 & 4,0 & 0,936 \\
\hline Elevado & 82 & 85,4 & 92,0 & 96,0 & \\
\hline \multicolumn{6}{|c|}{ Estadiamento clínico $(n=91)$} \\
\hline $\mathrm{ECl}$ & 26 & 26,1 & 21,7 & 22,7 & 0,702 \\
\hline EC2 & 65 & 73,9 & 78,3 & 77,3 & \\
\hline
\end{tabular}

Legendas: Baixo consumo: Consumo de alimentos ultraprocessados <p25; Alto consumo: Consumo de alimentos ultraprocessados $\geq \mathrm{p} 75$; Idade: Ponto de corte do Instituto Nacional de Câncer José Alencar Gomes da Silva (INCA, 2020). Sem companheiro: Viúva, divorciada, solteira. Estado Nutricional - Classificação para


$-\leq 27 \mathrm{~kg} / \mathrm{m}^{2}$; Sobrepeso: IMC $>27 \mathrm{~kg} / \mathrm{m}^{2}$. Baixo/moderado risco cardiometabólico: $\mathrm{CC}<88 \mathrm{~cm}$; Elevado risco cardiometabólico: CC $\geq 88 \mathrm{~cm}$. EC1: Estadiamentos clínicos 0, I e II; EC2: Estadiamentos clínicos III e IV.

para o aumento de peso e a instalação da obesidade; o maior consumo de alimentos açucarados tem relação direta com maiores índice e carga glicêmica, aumento da glicemia, hiperinsulinemia e cascatas metabólicas que favorecem a carcinogênese.

Em pacientes brasileiras sobreviventes do câncer de mama, estudo recente de Alves et al. ${ }^{3}$ mostrou que 14\% das calorias ingeridas por essas mulheres são provenientes de alimentos ultraprocessados; entretanto, esse consumo não teve associação com estado nutricional. Nestes achados, o consumo de ultraprocessados das mulheres sobreviventes do câncer de mama é maior e responde por $27,1 \%$ do total de calorias ingeridas e se assemelha ao encontrado para a população brasileira em 2017 $(21,5 \%)^{24}$. Essa sinergia de resultados sinaliza que, após o diagnóstico de câncer de mama, as pacientes não mudam o seu consumo alimentar para o padrão mais saudável, apesar de estarem em um momento favorável para essas mudanças ${ }^{25,26}$.
A alimentação adequada tem sido posta pelo $\mathrm{WCRF}^{2}$ como recomendação para pacientes sobreviventes e apontada como aspecto modificável no sentido de prevenir recidiva da doença. Entretanto, para que essa qualidade no consumo alimentar exista entre mulheres sobreviventes do câncer de mama, há a necessidade de orientação nutricional adequada e acompanhamento desde o diagnóstico ${ }^{26,27}$. Em se tratando de extensão e propósito de processamento dos alimentos, Alves et al. ${ }^{3}$ levantam importante discussão sobre a necessidade de um ponto de corte que indique o que significa um consumo seguro de ultraprocessados. Os autores destacam que essa fragilidade da inexistência de um ponto de corte dificulta as associações entre o consumo de ultraprocessados e o câncer de mama e a construção de orientaçôes alimentares para a população.

Apesar de ainda náo se ter esse ponto de corte, estes achados mostram que o maior consumo de ultraprocessados esteve associado com reduçáo na ingestão 
Tabela 2. Média do consumo absoluto e relativo de alimentos, segundo calorias/dia e a extensão e o propósito de processamento dos alimentos

\begin{tabular}{|c|c|c|c|c|c|}
\hline $\begin{array}{l}\text { Extensão e propósito } \\
\text { de processamento dos } \\
\text { alimentos }\end{array}$ & Kcal/dia & $\begin{array}{c}\text { \% da } \\
\text { ingestão } \\
\text { no total de } \\
\text { energia }\end{array}$ & $\begin{array}{l}\text { Extensão e propósito } \\
\text { de processamento dos } \\
\text { alimentos }\end{array}$ & Kcal/dia & $\begin{array}{c}\text { \% da } \\
\text { ingestão } \\
\text { no total de } \\
\text { energia }\end{array}$ \\
\hline \multirow{3}{*}{$\begin{array}{l}\text { Alimentos in natura } \\
\text { ou minimamente } \\
\text { processados e } \\
\text { ingredientes culinários }\end{array}$} & \multirow{3}{*}{$1.590,7$} & \multirow{3}{*}{69,8} & Carne salgada & 7,7 & 0,3 \\
\hline & & & Bebidas $^{b}$ & 4,6 & 0,2 \\
\hline & & & $\begin{array}{l}\text { Alimentos } \\
\text { ultraprocessados }\end{array}$ & 617,5 & 27,1 \\
\hline Carnes e ovos ${ }^{a}$ & 308,9 & 13,6 & Pães industrializados & 196,9 & 8,6 \\
\hline Frutas & 211,9 & 9,3 & Biscoitos $^{c}$ & 76,5 & 3,4 \\
\hline Leite & 154,0 & 6,8 & Embutidos & 57,4 & 2,5 \\
\hline Arroz & 179,7 & 7,9 & Sorvete & 42,4 & 1,9 \\
\hline Azeite & 99,9 & 4,4 & Bolo & 41,2 & 1,8 \\
\hline Vitamina de leite & 87,7 & 3,8 & \multirow{2}{*}{$\begin{array}{l}\text { logurte adicionado de } \\
\text { açúcar }\end{array}$} & \multirow{2}{*}{29,8} & \multirow{2}{*}{1,3} \\
\hline Feijão e ervilha & 81,8 & 3,6 & & & \\
\hline Café & 84,8 & 3,7 & Requeijão & 28,0 & 1,2 \\
\hline Suco de fruta & 72,1 & 3,2 & Refrigerantes & 25,1 & 1,1 \\
\hline Cuscuz & 69,0 & 3,0 & Margarina & 24,7 & 1,1 \\
\hline Batata e aipim & 61,6 & 2,7 & Lasanha & 23,9 & 1,1 \\
\hline Farinha & 50,0 & 2,2 & Pastelaria & 22,2 & 1,0 \\
\hline Aveia & 41,5 & 1,8 & Pizza & 14,5 & 0,6 \\
\hline Verduras e legumes & 29,7 & 1,3 & Batata frita & 11,3 & 0,5 \\
\hline Sopas & 27,3 & 1,2 & Chocolates, bombons & 10,2 & 0,4 \\
\hline Açúcar & 20,6 & 0,9 & Salada de maionese & 7,9 & 0,3 \\
\hline Manteiga & 10,0 & 0,4 & Maionese & 3,9 & 0,2 \\
\hline Alimentos processados & 70,3 & 3,1 & Adoçante & 1,1 & 0,0 \\
\hline Frutas em conserva & 36,8 & 1,6 & Catchup & 0,5 & 0,0 \\
\hline Queijo & 13,1 & 0,6 & Total & $2.278,5$ & 100,0 \\
\hline Enlatados milho e ervilha & 8,0 & 0,3 & inho; ': Inclui biscoitos recheados, & $\begin{array}{l}\text { rco, frango e v } \\
\text { es e salgados. }\end{array}$ & ; b: Inclui \\
\hline
\end{tabular}

Tabela 3. Média de ingestão de nutrientes segundo o consumo de ultraprocessados

\begin{tabular}{lcccc}
\hline \multirow{2}{*}{ Nutriente } & Total & $\begin{array}{c}\text { Baixo consumo de } \\
\text { ultraprocessados }\end{array}$ & $\begin{array}{c}\text { Alto consumo de } \\
\text { ultraprocessados }\end{array}$ & p \\
\cline { 2 - 4 } & Média & Média & Média \\
\hline Carboidrato (g) & 322,1 & 322,1 & 317,8 & 0,749 \\
Proteína (g) & 84,4 & 90,5 & 80,5 & 0,037 \\
Lipídio (g) & 60,1 & 56,7 & 62,4 & 0,185 \\
Gordura saturada (g) & 20,0 & 19,5 & 20,6 & 0,525 \\
Gordura monoinsaturada (g) & 18,8 & 16,8 & 18,9 & 0,141 \\
Gordura poli-insaturada (g) & 9,3 & 8,3 & 11,0 & 0,002 \\
Fibra (g) & 31,5 & 35,5 & 30,8 & 0,045 \\
Sódio (mg) & $1.707,4$ & $1.478,1$ & $1.874,1$ & 0,007 \\
\hline
\end{tabular}

Legendas: Baixo consumo: Consumo de alimentos ultraprocessados $<$ p25; Alto consumo: Consumo de alimentos ultraprocessados $\geq \mathrm{p} 75$; Diferença de médias por t-Student. Significância $\mathrm{p}<0,05$. 
Tabela 4. Coeficiente de regressão linear $(\beta)$ da relação entre ingestão de ultraprocessados e ingestão de energia e de nutrientes específicos

\begin{tabular}{|c|c|c|c|c|c|c|}
\hline & $\beta^{a}$ & $\mathbf{P}$ & $\beta^{\mathbf{b}}$ & $\mathbf{p}$ & $\beta^{c}$ & $\mathbf{P}$ \\
\hline Alimentos in natura, Kcal* & $-0,794$ & 0,008 & $-0,835$ & 0,005 & $-0,800$ & 0,008 \\
\hline Alimentos açucarados, Kcal & $-0,426$ & 0,054 & $-0,459$ & 0,044 & $-0,404$ & 0,080 \\
\hline Proteínas, \%VET* & 0,022 & 0,273 & 0,022 & 0,253 & 0,031 & 0,111 \\
\hline Gorduras, \%VET & 0,039 & 0,039 & 0,039 & 0,047 & 0,045 & 0,021 \\
\hline Gordura saturada, \%VET & 0,011 & 0,123 & 0,011 & 0,133 & 0,014 & 0,047 \\
\hline Gordura monoinsaturada, \%VET & 0,016 & 0,041 & 0,014 & 0,076 & 0,016 & 0,039 \\
\hline Gordura poli-insaturada, \%VET & 0,018 & $<0,0001$ & 0,018 & $<0,0001$ & 0,019 & $<0,0001$ \\
\hline Fibra, $\mathrm{g}^{*}$ & $-0,006$ & 0,408 & $-0,007$ & 0,282 & $-0,007$ & 0,318 \\
\hline Sódio, mg* & 0,905 & 0,077 & 0,890 & 0,083 & 108,78 & 0,035 \\
\hline
\end{tabular}

Legendas: Regressão linear: ${ }^{a}$ Modelo bruto; ${ }^{b}$ Modelo ajustado por idade e escolaridade; ${ }^{c}$ Ajustado por idade, escolaridade e IMC: Índice de massa corporal. *Kcal: Quilocalorias, VET: Valor energético total, g: Gramas, mg: Miligramas.

de in natura. Os alimentos in natura envolvem carnes, ovos, frutas e hortaliças ${ }^{22} \mathrm{e}$ os de origem vegetal têm característica protetoras para o processo de carcinogênese, como a presença de vitaminas antioxidantes, fibras e outros compostos bioativos ${ }^{28,29}$. Mulheres com câncer de mama que têm uma dieta prudente, rica em verduras e legumes apresentam menor risco de recidiva e morte pela doença ${ }^{30}$. Entre as pacientes deste estudo, as frutas responderam por apenas 9,3\% das calorias ingeridas e as hortaliças nem aparecem como escolhas alimentares. Um agravante desse achado é a menor ingestáo de fibra entre as mulheres com alto consumo de ultraprocessados, uma vez que as fibras auxiliam no gerenciamento do peso e da glicemia, ambos relacionados à promoção da carcinogênese.

Outro achado que merece atençấo é o aumento no consumo de gordura total e suas fraçóes quanto maior o consumo de ultraprocessados. Sabe-se que metabólitos relacionados ao consumo de manteiga, margarina e sobremesas, fontes de gordura saturada, colaboram com a etiologia do câncer de mama ${ }^{31,32}$.

Além do olhar sobre o consumo de vegetais e gorduras, entre essas pacientes, encontrou-se menor ingestão de proteínas naquelas com alto consumo de ultraprocessados. $\mathrm{O}$ consumo de proteínas entre pacientes sobreviventes do câncer é estratégia importante para manutenção de uma adequada composição corporal e minimiza o desenvolvimento de obesidade sarcopênica sabidamente envolvida com menor sobrevida livre da doença no câncer de mama $^{33}$. Os resultados deste estudo não trazem a associação entre o diagnóstico nutricional e o consumo de ultraprocessados, mas mostram elevada prevalência de excesso de peso e CC, os quais têm associação com risco de câncer ou piora do estado clínico das pacientes.

Os resultados aqui apresentados apontam que as mulheres sobreviventes do câncer têm um perfil de consumo de ultraprocessados de produtos ricos em amido e farinha branca, seguidos das carnes processadas, produtos açucarados e gorduras. Segundo Fiolet et al. ${ }^{5}$, entre os alimentos ultraprocessados mais consumidos, estáo os produtos açucarados, seguidos de bebidas e alimentos ricos em amidos e cereais matinais. No Brasil, os alimentos ultraprocessados mais presentes na alimentação nacional (frios e embutidos, biscoitos doces e salgados, margarina, bolos e tortas doces, pães, doces em geral, bebidas adoçadas carbonatadas e chocolate) coincidem com os encontrados neste estudo ${ }^{24}$.

Quanto ao consumo de alimentos açucarados (pão industrializados, biscoitos, sorvetes, bolos e refrigerantes), verificou-se que $16,7 \%$ do total de calorias ingeridas pelas pacientes sáo provenientes desses tipos de alimentos. Segundo a $\mathrm{OMS}^{34}$, no máximo $10 \%$ das calorias diárias devem vir do consumo de açúcar, e a WCRF² recomenda limitar ao máximo esse consumo. Moubarac et al. ${ }^{35}$ encontraram que as bebidas ultraprocessadas (incluindo bebidas gaseificadas, sucos de fruta e bebidas de frutas) e alimentos ultraprocessados adoçados (incluindo doces, biscoitos, bolos, sobremesas, produtos lácteos) representaram cerca de $20 \%$ do consumo diário de calorias de canadenses, valor semelhante ao encontrado nesta pesquisa.

Luiten et al. ${ }^{36}$ observaram uma correlação positiva entre a extensão e o propósito de processamento industrial com a classificaçáo dos nutrientes, constatando que os alimentos ultraprocessados apresentaram pior perfil nutricional entre todos os grupos analisados. Diante disso, a recomendação do Guia Alimentar para a remoção desses alimentos do dia a dia da população é incontestável para uma melhor escolha alimentar e prevenção das doenças crônicas não transmissíveis. A WCRF ${ }^{2}$ também apresenta como uma das suas recomendações para prevenção do câncer e sua recidiva limitar o consumo de ultraprocessados. Uma elevação de $10 \%$ na proporçáo do consumo de alimentos ultraprocessados na dieta está relacionada a aumentos significativos no risco de câncer total (12\%) e no risco de câncer de mama $(11 \%)^{5}$. 
Apesar de este estudo não ser de seguimento e não apresentar uma relaçáo de causalidade entre o consumo de ultraprocessados e o desenvolvimento do câncer de mama, afirmar-se a relevância dos resultados por indicarem que, mesmo após o diagnóstico de câncer de mama, o consumo de ultraprocessados permanece elevado. Além disso, a relação desse consumo com outros nutrientes envolvidos com promoção e proteção da carcinogênese chama atenção para a necessidade do desenvolvimento de estratégias específicas para o grupo de mulheres sobreviventes ao câncer de mama. Outro aspecto positivo deste estudo é a amostra exclusiva de pacientes sobreviventes do câncer, população com a qual os estudos alimentares são escassos. Destaca-se, ainda, como ponto forte, a contribuição desses achados para o desenvolvimento de protocolos clínicos de acompanhamento de pacientes sobreviventes do câncer de mama, além de favorecer discussóes sobre políticas públicas direcionadas para pacientes oncológicos nas diferentes categorias de sobreviventes ${ }^{37}$.

\section{CONCLUSÃO}

Pacientes sobreviventes do câncer de mama têm quase um terço da sua ingestáo de energia proveniente de alimentos ultraprocessados e o consumo desses alimentos implica em redução no consumo de in natura e no aumento na ingestão de gordura total e sódio. Esse consumo alimentar pode contribuir para a obesidade, fator de risco para recidiva da doença. Esses achados indicam a necessidade de melhorar o consumo alimentar de mulheres sobreviventes do câncer de mama, a fim de garantir a manutenção de um peso saudável e, desse modo, minimizar o risco de recidiva.

\section{CONTRIBUIÇÕES}

Julianne do Nascimento Sales contribuiu na concepçáo e/ou no planejamento do estudo; na obtenção, análise e interpretação dos dados; e na redação. Manuella Cunha Barbosa contribuiu na concepçáo e/ou no planejamento do estudo; na obtenção, análise e interpretação dos dados. Ilana Nogueira Bezerra contribuiu na análise e interpretação dos dados; e na revisão crítica. Sara Maria Moreira Lima Verde contribuiu na concepção e/ou planejamento do estudo; na interpretação dos dados; e na redação e revisão crítica. Todas as autoras aprovaram a versão final a ser publicada.

\section{AGRADECIMENTOS}

Ao Centro de Estudos do Centro Regional Integrado de Oncologia pela abertura para realizaçáo da coleta de dados; às pacientes que participaram do estudo por seu Consentimento Livre e Esclarecido; à Priscila Carmelita Paiva Dias Carneiro, nutricionista e professora da Unifor pelo apoio à realização do estudo.

\section{DECLARAÇÃO DE CONFLITO DE INTERESSES}

Nada a declarar.

\section{FONTES DE FINANCIAMENTO}

Não há.

\section{REFERÊNCIAS}

1. Blanco-Rojo R, Sandoval-Insausti H, López-Garcia E, et al. Consumption of ultra-processed foods and mortality: a national prospective cohort in Spain. Mayo Clin Proc. 2019;94(11):2178-88. doi: https://doi.org/10.1016/j. mayocp.2019.03.035

2. World Cancer Research Fund; American Institute for Cancer Research. Recommendations and public health and policy implications [Internet]. London: WCRF; 2018 May [cited 2020 May 9]. Available from: https:// www.wcrf.org/sites/default/files/Cancer-Prevention Recommendations 2018.pdf

3. Alves PC, Sampaio HAC, Henriques EMV, et al. Dietary assessment of women surviving breast cancer according to the dietary guidelines for the Brazilian population. Rev Nutr. 2019;32:e180054. doi: https:// doi.org/10.1590/1678-9865201932e190054

4. Monge A, Lajous M. Ultra-processed foods and cancer. BMJ. 2018;360:k599. doi: https://doi.org/10.1136/ bmj.k599

5. Fiolet T, Srour B, Sellem L, et al. Consumption of ultraprocessed foods and cancer risk: results from NutriNetSanté prospective cohort. BMJ. 2018;360:k322. doi: https://doi.org/10.1136\%2Fbmj.k322

6. Monteiro CA, Cannon G, Moubarac JC, et al. The UN decade of nutrition, the NOVA food classification and the trouble with ultra-processing. Public Health Nutr. 2018;21(1):5-17. doi: https://doi.org/10.1017/ S1368980017000234

7. Mendonça RD, Pimenta AM, Gea A, et al. Ultraprocessed food consumption and risk of overweight and obesity: the University of Navarra Follow-up (SUN) cohort study. Am J Clin Nutr. 2016;104(5):1433-40. doi: https://doi. org/10.3945/ajcn.116.135004

8. Tumminia A, Vinciguerra F, Parisi M, et al. Adipose tissue, obesity and adiponectin: role in endocrine cancer risk. Int J Mol Sci. 2019;20(12):2863 doi: https://doi. org/10.3390/ijms20122863

9. Gultekin F, Yasar S, Gurbuz N, et al. Food additives of public concern for their carcinogenicity. J Nutrition 
Health Food Sci. 2015;3(4):1-6 doi: https://doi. org/10.15226/jnhfs.2015.00149

10. Poulsen R, Cedergreen N, Hayes T. Nitrate: an environmental endocrine disruptor? A review of evidence and research needs. Environ Sci Technol. 2018;52(7):3869-87. doi: https://doi.org/10.1021/acs. est.7b06419

11. International Agency for Research on Cancer [Internet]. Lyon, France: Global Cancer; 2018. Estimated number of prevalent cases (5-year) worldwide, females, all ages; 2018 [cited 2019 Nov 6]; [about 1 screen]. Available from: https://gco.iarc.fr/today/onlineanalysis-multi-bars? $\mathrm{v}=2018 \&$ mode $=$ cancer $\&$ mode_po pulation $=$ countries $\&$ population $=900 \&$ populations $=900 \& \mathrm{key}=$ total $\&$ sex $=2 \&$ cancer $=39 \&$ type $=0 \&$ stati stic $=5 \&$ prevalence $=0 \&$ population_group $=0 \&$ ages_ group $\% 5 \mathrm{~B} \% 5 \mathrm{D}=0 \&$ ages_group $\% 5 \mathrm{~B} \% 5 \mathrm{D}=17 \& \mathrm{nb}$ it e m s $=10 \&$ grou _ _ ancer $=1 \&$ include_ $\mathrm{nmsc}=1 \&$ include_nmsc_other $=1 \&$ type_multiple= $\% 257$ B $\% 2522$ inc $\% 2522 \% 253$ Afalse $\% 252$ C\% 252 2 mort $\% 2522 \% 253$ Afalse $\% 252 \mathrm{C} \% 2522$ prev\% 252 2\%253Atrue $\% 257$ D\&orientation=horizontal \&ty pe_sort=0\&type_nb_items=\%257B $\% 2522$ top $\% 252$ 2\%253Atrue $\% 252$ C\%2522bottom $\% 2522 \% 253$ Afa 1se\%257D\&population_group_globocan_id=

12. Latino-Martel P, Cottet V, Druesne-Pecollo N, et al. Alcoholic beverages, obesity, physical activity and other nutritional factors, and cancer risk: a review of the evidence. Crit Rev Oncol Hematol. 2016;99:308-23. doi: https://doi.org/10.1016/j.critrevonc.2016.01.002

13. Pan H, Gray R, Braybrooke J, et al. 20-year risks of breast-cancer recurrence after stopping endocrine therapy at 5 years. N Engl J Med. 2017;377:1836-46. doi: https://doi.org/10.1056/NEJMoa1701830

14. Instituto Nacional de Câncer José Alencar Gomes da Silva. Posicionamento do Instituto Nacional de Câncer José Alencar Gomes da Silva acerca do sobrepeso e obesidade. Rio de Janeiro: INCA; 2017.

15. Mullan F. Seasons of survival: reflections of a physician with cancer. N Engl J Med. 1985;313:270-3. doi: https:// doi.org/10.1056/NEJM198507253130421

16. Dias MCG, Horie LM, Waitzberg DL. Exame físico e antropometria. In: Waitzberg DL. Nutrição oral, enteral e parenteral na prática clínica. 4. ed. São Paulo: Atheneu; 2002. p. 383-419.

17. World Health Organization. Obesity: preventing and managing the global epidemic. Report of a WHO Consultation [Internet]. Geneva: WHO; 2000 [cited 2020 May 9]. 268 p. (WHO Technical Report Series, 894). Available from: https://http://apps.who.int/ iris/bitstream/10665/42330/1/WHO_TRS_894. pdf?ua $=1 \& u a=1$

18. Lipschitz DA. Screening for nutritional status in the elderly. Prim Care. 1994;21(1):55-67.
19. World Health Organization. Preparation and use of foodbased dietary guidelines. Report of a Joint FAO/ WHO Consultation [Internet]. Geneva: WHO; 1998 [cited 2020 May 9]. Available from: https:// https:// apps.who.int/iris/bitstream/handle/10665/42051/ WHO_TRS_880.pdf;jsessionid=9E2CAE503CD00A 8051D98AA767263323? sequence $=1$

20. Associação Brasileira para o Estudo da Obesidade e da Síndrome Metabólica. Diretrizes brasileiras de obesidade [Internet]. 4. ed. São Paulo: ABESO; 2016 [acesso 2020 maio 9]. Disponível em: https://abeso.org.br/ wp-content/uploads/2019/12/Diretrizes-DownloadDiretrizes-Brasileiras-de-Obesidade-2016.pdf

21. Lima FEL, Slater B, Latorre MRDO, et al. Validade de um questionário quantitativo de frequência alimentar desenvolvido para população feminina no nordeste do Brasil. Rev Bras Epidemiol. 2007;10(4):483-90. doi: http://doi.org/10.1590/S1415-790X2007000400006

22. Monteiro CA, Cannon G, Levy R, et al. NOVA. The star shines bright: food classification. Public health. World Nutrition. 2016;7(1-3):28-38.

23. Willett W. Nutritional epidemiology. 3. ed. New York: Oxford University Press; 2013. (Monographs in epidemiology and biostatistics; v. 40).

24. Louzada MLC, Martins APB, Canella DS, et al. Alimentos ultraprocessados e perfil nutricional da dieta no Brasil. Rev Saúde Pública. 2015;49:38. doi: https:// doi.org/10.1590/S0034-8910.2015049006132

25. Custódio IDD, Marinho EC, Gontijo CA, et al. Impact of chemotherapy on diet and nutritional status of women with breast cancer: a prospective study. Plos One. 2016;11(6):e0157113. doi: https://doi.org/10.1371/ journal.pone. 0157113

26. Custódio IDD, Franco FP, Marinho EC, et al. Prospective analysis of food consumption and nutritional status and the impact on the dietary inflammatory index in women with breast cancer during chemotherapy. Nutrientes. 2019;11(11):2610. doi: https://doi.org/10.3390/ nu11112610

27. Ferreira IB, Marinho EC, Custódio IDD, et al. Consumo alimentar e estado nutricional de mulheres em quimioterapia. Ciênc Saúde Coletiva. 2016;21(7):2209-18. doi: https://doi.org/10.1590/141381232015217.05412015

28. Amiot-Carlin MJ. [Fruit and vegetable consumption: what benefits, what risks?]. Rev Prat. 2019;69(2):13942. French.

29. Poirier AE, Ruan Y, Hebert LA, et al. Estimates of the current and future burden of cancer attributable to low fruit and vegetable consumption in Canada. Prev Med. 2019;122:20-30. doi: https://doi.org/10.1016/j. ypmed.2019.03.013

30. Kaledkiewicz E, Szostak-Węgierek D. Dietary practices and nutritional status in survivors of breast cancer. Rocz Panstw Zakl Hig. 2018;69(2):175-82. 
31. Playdon MC, Zielgler RG, Sampson JN, et al. Nutritional metabolomics and breast cancer risk in a prospective study. Am J Clin Nutr. 2017;106(2):637-49. doi: https:// doi.org/10.3945/ajcn.116.150912

32. Xia H, Ma S, Wang S, et al. Meta-analysis of saturated fatty acid intake and breast cancer risk. Medicine (Baltimore). 2015;94(52):e2391. doi: https://doi. org/10.1097/MD.0000000000002391

33. Carneiro IP, Mazurak VC, Prado CM. Clinical implications of sarcopenic obesity in cancer. Curr Oncol Rep. 2016;18(62). doi: https://doi.org/10.1007/s11912016-0546-5

34. World Health Organization. Guideline: sugars intake for adults and children [Internet]. Geneva: WHO; 2015 [cited 2017 Dec 4]. Available from: http://https://apps. who.int/iris/rest/bitstreams/668769/retrieve

35. Mourabac JC, Batal M, Louzada ML, et al. Consumption of ultra-processed foods predicts diet quality in Canada. Appetite. 2017;108:512-20. doi: https://doi. org/10.1016/j.appet.2016.11.006

36. Luiten CM, Steenhuis IH, Eyles H, et al. Ultra-processed foods have the worst nutrient profile, yet they are the most available packaged products in a sample of New Zealand supermarkets. Public Health Nutr. 2016;19(3):530-8. doi: https://doi.org/10.1017/S1368980015002177

37. Surbone A, Tralongo P. Categorization of cancer survivors: why we need it. J Clin Oncol. 2016;34(28):3372-4. doi: https://doi.org/10.1200/jco.2016.68.3870

Recebido em 9/6/2020

Aprovado em 28/7/2020 